\title{
Hepatic steatosis in Cushing's syndrome: a radiological assessment using computed tomography
}

A G Rockall, S A Sohaib, D Evans, G Kaltsas ${ }^{1}$, A M Isidori ${ }^{1}$, J P Monson ${ }^{1}$, G M Besser ${ }^{1}$, A B Grossman ${ }^{1}$ and R H Reznek

Departments of Diagnostic Radiology and ${ }^{1}$ Endocrinology, St Bartholomew's Hospital, West Smithfield, London EC1A 7BE, UK

(Correspondence should be addressed to A G Rockall, Department of Diagnostic Radiology, St Bartholomew's Hospital, Dominion House,

St Bartholomew's Close, West Smithfield, London EC1A 7BE, UK; Email: a.g.rockall@qmul.ac.uk)

\begin{abstract}
Objective: Hepatic steatosis may occur in association with insulin resistance and obesity, two features commonly seen in Cushing's syndrome (CS). The aim of this report is to assess the prevalence of hepatic steatosis in patients with active CS using computed tomography (CT) and to identify any associations between hepatic steatosis, endocrine and biochemical variables and body fat distribution. Patients and measurements: We identified 50 patients with active CS in whom appropriate CT was available to allow measurement of liver and spleen attenuation. In 26 patients, abdominal fat measurements were also available. Serum markers of CS and liver function tests were recorded.

Results: Ten of 50 patients had a liver-to-spleen CT attenuation ratio (L/S) of less than 1 , indicating hepatic steatosis. There was a significant negative correlation between both liver attenuation and $\mathrm{L} / \mathrm{S}$ ratio with total abdominal fat area, visceral fat area, the percentage of visceral fat and the visceral to subcutaneous fat ratio; the strongest negative correlation was found between visceral fat area and $\mathrm{L} / \mathrm{S}$ ratio $(r=-0.638, P<0.001, n=26)$. L/S ratio positively correlated with alkaline phosphatase levels $(r=+0.423, P=0.044, n=23)$ but with no other serum marker of CS activity or liver enzyme. Conclusions: We have demonstrated hepatic steatosis on CT in $20 \%$ of patients with active CS. The presence of hepatic steatosis was significantly correlated with total abdominal fat area and visceral fat area.
\end{abstract}

European Journal of Endocrinology 149 543-548

\section{Introduction}

Non-alcoholic fatty liver disease (NAFLD) is a clinicopathological entity that is increasingly recognised as a possible precursor to cirrhosis and is now the second or third commonest liver disease in hepatology outpatient practice in North America (1). NAFLD encompasses a range of pathological abnormalities, including simple hepatic steatosis, steatonecrosis and steatohepatitis $(2,3)$. The most severe form of NAFLD is non-alcoholic steatohepatitis or NASH. This term was coined in 1980 to describe pathological changes that are indistinguishable from the features most commonly seen in alcoholic liver disease but with no history of alcohol misuse (4). In NAFLD, several aetiological factors have been implicated: obesity, type II diabetes, malnutrition, hyperlipidaemia and various medications (corticosteroids, synthetic oestrogens, amiodarone, nifedipine) have all been associated $(3,4)$. NAFLD is often first suggested from the results of liver function tests at routine health screening, as patients are usually asymptomatic.

Non-alcoholic, simple hepatic steatosis is generally non-progressive, but between $2.5-4 \%$ progress to cirrhosis $(5,6)$. However, in patients referred for the investigation of persistently abnormal liver function tests, with no evidence of viral hepatitis or of alcohol use, $15-50 \%$ of patients are found to have fibrosis or cirrhosis (7), and a long-term follow-up study of patients with NASH has reported that $22 \%$ of patients had developed cirrhosis after 10 years (6).

It is the experience within our department that patients with Cushing's syndrome are often noted to have the appearances of fatty change on ultrasound and computed tomography (CT) scans of the liver. However, the incidence of NAFLD in Cushing's syndrome is unknown, nor is it known whether its occurrence is related to the patient's obesity or body fat distribution or due to the altered hormonal milieu. An increased prevalence of NAFLD in Cushing's syndrome could potentially increase the risk of NASH, with the associated increase in morbidity and mortality due to the risk of developing cirrhosis.

A strong correlation has been reported between CT attenuation values of the liver and the presence of hepatic steatosis histologically (8). The presence of hepatic steatosis may be quantified non-invasively using CT, with a good correlation between liver 
attenuation and histological and biochemical estimates of liver lipid concentration (8, 9). A CT liver attenuation less than spleen attenuation has also been shown to be a reliable indicator of fatty liver $(9,10)$. Thus, CT may provide a non-invasive test for identifying hepatic steatosis, although CT cannot differentiate between simple steatosis and steatohepatitis.

In this study, our aims were to document the prevalence of hepatic steatosis in patients with active Cushing's syndrome using CT, and to identify any correlation between hepatic steatosis and body fat distribution, serum markers of Cushing's syndrome and liver function tests.

\section{Methods}

\section{Scan technique and measurement of liver attenuation}

Patients were scanned in the supine position using a GE 9800 scanner up to 1993 and a GE Hi-Speed Adv RP (General Electric, Milwaukee, USA) scanner from 1993 onwards. A single $10 \mathrm{~mm}$ slice obtained prior to intravenous administration of contrast medium through the right lobe of the liver was used for analysis. Patients with scans performed only after contrast medium injection were excluded from liver analysis. A $100 \mathrm{~mm}^{2}$ region of interest (ROI) was placed on five areas of this section and the Hounsfield units of each ROI were recorded (Fig. 1). Care was taken not to include any vessels in the ROIs. The sections were viewed on liver window settings. The mean liver attenuation was calculated from the five liver ROIs. The attenuation of the spleen was measured in the

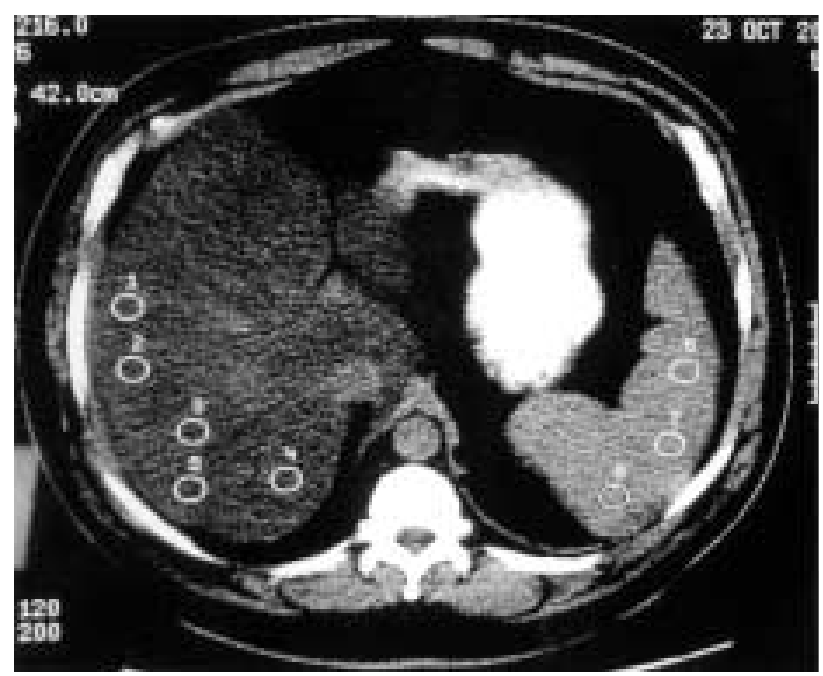

Figure 1 CT scan through the liver and spleen, pre-contrast enhancement. Five regions of interests (ROls, indicated as white circles) are drawn on the liver (numbers 1-5) and three on the spleen (numbers 6-8). The liver attenuation is the mean of the five ROls. The spleen attenuation is the mean of the three ROIs. same way, but using only three ROIs, reflecting the smaller size of the spleen. The CT attenuation characteristics of the liver were recorded as: 1) the mean liver attenuation, 2) the liver-to-spleen ratio (L/S ratio) and 3) as categorical data, being either fatty (with an $\mathrm{L} / \mathrm{S}$ ratio of $<1$ ) or not fatty (with an $\mathrm{L} / \mathrm{S}$ ratio of greater than or equal to 1$)(8-10)$.

\section{Patients}

All patients with newly diagnosed Cushing's syndrome in our institution undergo CT scanning of the adrenal glands as part of our standard protocol. Fifty-one patients ( 42 female) with active Cushing's syndrome were identified with non-contrast CT of the liver between 1989 and 2001. None had a history of excess alcohol intake, defined as $>21$ units per week for women or $>28$ units per week for men. One of the patients had had a splenectomy, and thus the L/S ratio could not be calculated in this case. Eight other patients with appropriate CT scans were excluded due to either a significant history of alcohol ingestion or if they had been treated with the cortisol lowering drugs ketoconazole or metyrapone for more than 3 months. The mean age of the 50 patients included in the analysis was 46.6 years (range 14 to 79 years). The diagnosis of Cushing's syndrome was made by the finding of elevated midnight cortisol levels when asleep (normal values $<50 \mathrm{nmol} / \mathrm{l}$ ) and the loss of suppression to levels less than $50 \mathrm{nmol} / \mathrm{l}$ $48 \mathrm{~h}$ after $2 \mathrm{mg}$ dexamethasone given 6 hourly for $48 \mathrm{~h}$ as a suppression test; Cushing's disease was based on a detectable $0900 \mathrm{~h}$ plasma adrenocorticotrophin (ACTH) concentration and either two dynamic tests compatible with the diagnosis or a positive central-to-peripheral gradient on bilateral petrosal sinus sampling (11). The diagnosis was considered as confirmed if there was positive histology of a corticotroph adenoma, adrenal tumour or ectopic source, or if there was clinical and biochemical resolution following treatment (11). Thirty-nine patients had Cushing's disease, six had adrenocortical adenomata, four had a confirmed ectopic source of ACTH, whilst in two patients the cause of Cushing's syndrome remains unknown. The body mass index $\left(\mathrm{BMI}=\right.$ weight in $\mathrm{kg} /$ height in $\left.\mathrm{m}^{2}\right)$ in men $(n=9)$ was $25.7 \pm 3.3$, range 19.9-30.5 (mean \pm one standard deviation) and in women $(n=39)$ was $29.3 \pm 7.2$, range $20.8-50.6$.

\section{Fat distribution analysis}

Twenty-six patients were identified in whom the subcutaneous and visceral abdominal fat distribution could also be measured. A single $10 \mathrm{~mm}$ thick slice at the L4 vertebral level was used for fat analysis. Patients were excluded from the fat distribution analysis if the entire skin surface was not included in the field of view as, in some cases, the CT field of view was reduced to include only the adrenal glands and 
the retroperitoneum. Using GE software, the total fat area and visceral fat area ROIs were delineated by manually tracing a contour of each region, as demonstrated in Fig. 2. Fat pixels and therefore fat area were identified with threshold attenuation values between -50 to -250 Hounsfield units, as described by Borkan et al. (12). The subcutaneous fat area was then calculated by subtracting visceral from total fat area. The fat distribution was expressed as: (i) total, visceral and subcutaneous fat area; (ii) the ratio of visceral fat to total fat (\% visceral fat); and (iii) the ratio of visceral to subcutaneous fat (V:S ratio).

\section{Hormonal and biochemical data}

The results of endocrine investigations and liver function tests within 4 weeks of the CT date were obtained, including mean serum cortisol levels derived from five samples obtained throughout a single day (11), basal plasma ACTH levels (at $0900 \mathrm{~h}$ ), fasting glucose, fasting lipids and liver function tests (liver enzymes and bilirubin).

\section{Statistical analysis}

Data are expressed as means \pm one standard deviation. Fisher's exact test was applied to evaluate significant differences between frequency distributions. The difference of means between two groups was assessed by Student's $t$-test. Significance was taken as $P<0.05$. Spearman's correlation was applied to assess correlations between continuous variables. SPSS version 8 was used for statistical analysis (V. 8, SPSS Inc, Chicago, IL, USA).

\section{Results}

Of the total patient group, 10 of $50(20 \%)$ of the patients had hepatic steatosis (L/S ratio of less than 1 ). Patients with hepatic steatosis were older than those with no hepatic steatosis (Table 1). There was no significant difference in the prevalence of hepatic steatosis between male and female patients (Table 1). In the group of patients with hepatic steatosis, there was a greater proportion of patients with impaired glucose tolerance or diabetes ( 4 of $7,57 \%$ ) than in the group with no hepatic steatosis ( 8 of $33,24 \%$ ), but this did not reach statistical significance.

Table 2 gives the results of the mean liver attenuation values, the mean $\mathrm{L} / \mathrm{S}$ ratios and the mean values of the biochemical markers, with the standard deviations, for the whole group and for the female and male patients separately.

Table 3 details the results of Spearman's correlation coefficient of the liver attenuation and L/S ratio with the measured biochemical markers. The L/S ratio was positively correlated with alkaline phosphatase (ALP;

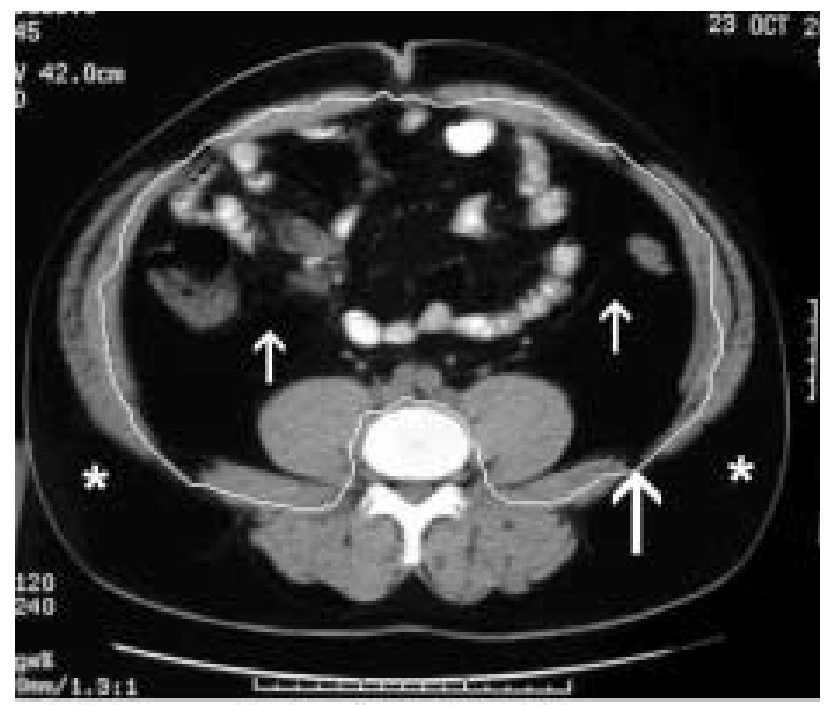

A

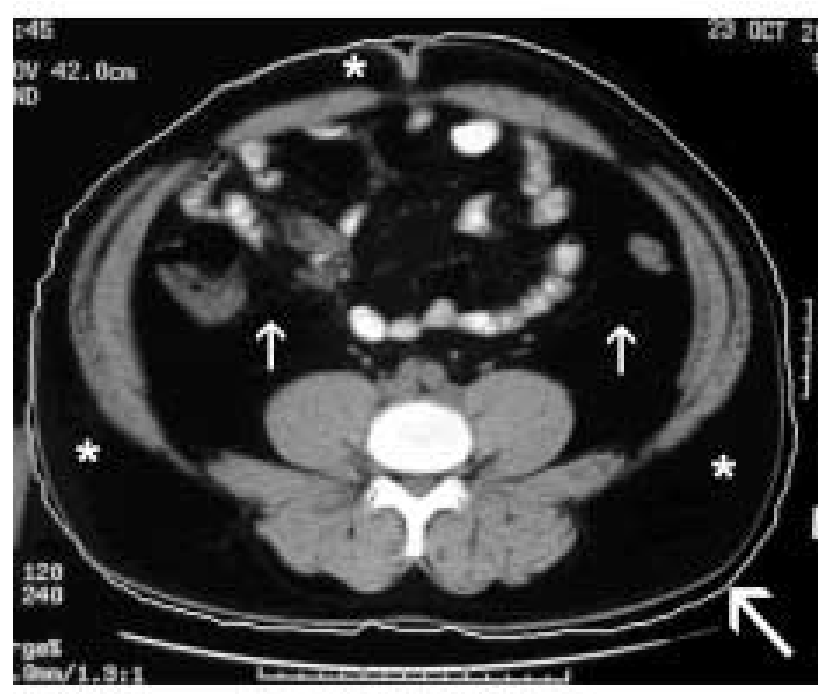

16

Figure 2 (A) CT scan at the level of $L 4$, with a region of interest (ROI) curve (hand-drawn white line, large arrow) delineating the region for measurement of visceral fat area. Small arrows, visceral fat; *, subcutaneous fat. (B) This is the same CT image as in (A) with a hand drawn region of interest (ROI) delineating the region for measurement of the total fat area (large arrow). The area of pixels having a Hounsfield unit of between -50 and -250 is then obtained. Subcutaneous fat $\left(^{*}\right)$ area is then calculated by subtraction of visceral fat (small arrows) area from total fat area.

$r=0.423, P=0.044, n=23)$ but with no other liver enzyme or serum marker of active Cushing's syndrome (Fig. 3).

Table 4 details the results of Spearman's correlation coefficient of liver attenuation and L/S ratio with body fat distribution. There was a strong negative correlation between both liver attenuation and L/S ratio with total fat, visceral fat, percentage of visceral fat and the $V: S$ ratio. The strongest negative correlation 
Table 1 Patient characteristics. Patients with a liver-to-spleen attenuation (L/S) ratio of equal to or greater than one are defined as not having hepatic steatosis and patients with an L/S ratio of less than one are defined as having hepatic steatosis.

\begin{tabular}{lccc}
\hline & $\begin{array}{c}\text { No hepatic } \\
\text { steatosis } \\
(\mathrm{L} / \mathrm{S}>/=1)\end{array}$ & $\begin{array}{c}\text { Hepatic } \\
\text { steatosis } \\
(\mathrm{L} / \mathrm{S}<1)\end{array}$ & $\begin{array}{c}\text { Significance } \\
\text { level }\end{array}$ \\
\hline Number & 40 & $10(20 \%)$ & \\
$\mathrm{M}: \mathrm{F}$ & $6: 34$ & $2: 8$ & $\mathrm{~N} / \mathrm{S}^{\mathrm{a}}$ \\
Age (years) & $44.5 \pm 16.5$ & $56.1 \pm 10.6$ & $P=0.04^{\star b}$ \\
BMI & $28.2 \pm 5.4$ & $29.2 \pm 8.9$ & $\mathrm{~N} / \mathrm{S}^{\mathrm{b}}$ \\
No diabetes vs & $25: 8$ & $3: 4$ & $\mathrm{~N} / \mathrm{S}^{\mathrm{a}}$ \\
IGT/diabetes & & &
\end{tabular}

${ }^{\star} P<0.05 ;{ }^{a}$ indicates Fisher's exact test (2-tailed); ${ }^{\mathrm{b}}$ indicates group $t$-test. $\mathrm{N} / \mathrm{S}$, not significant. IGT/diabetes, indicates the presence of impaired glucose tolerance or diabetes.

was between the L/S ratio and the visceral fat area $(r=-0.638, P<0.001, n=26)$. These results indicate that as the amount of visceral fat increases, hepatic steatosis increases.

\section{Discussion}

The prevalence of hepatic steatosis in the asymptomatic general population is not well documented in the published literature, with varying results from 3.5\% to $24 \%$, as described below $(13-17)$. Two postmortem studies of random deaths have reported on the prevalence of hepatic steatosis: in a study of 423 aircrew involved in fatal aircraft accidents, 66 cases (15\%) had a fatty liver, 11 of which had histopathological evidence of alcohol abuse (14); in a study of 503 fatal road traffic accidents, $24 \%$ had a fatty liver and fatty change was significantly correlated with body weight and increasing age (13). In neither of these studies was detailed information concerning alcohol misuse available. These populations may not be entirely representative of the general population as, particularly in the case of road traffic accidents, the level of alcohol use may have been a factor in the prevalence of fatty change.

In unselected patients undergoing annual medical check-ups in Japan, 105 of 3000 (3.5\%) were found to have a 'bright' liver on ultrasound, suggesting hepatic steatosis (15). An overall prevalence of $14 \%$ was reported on ultrasound appearances in a group of 2574 unselected residents of a district in Japan, with an increase in prevalence seen with age and alcohol consumption (16). In a large cohort study in Italy, $17.5 \%$ (1211 of 6917 ) of the population were found to have a clinical or biochemical sign to suggest chronic liver disease and, in this selected group, ultrasound was performed and found to demonstrate signs of hepatic steatosis in most of the group (18). The exact prevalence of hepatic steatosis on ultrasound was not given. However, the diagnosis of hepatic steatosis on ultrasound is subjective and not readily quantifiable.

CT has been shown to be a reliable and reproducible tool for the non-invasive quantification of hepatic steatosis $(8,9)$. However, the definition of hepatic steatosis on CT varies in the literature. The reliability of absolute attenuation values for normal liver is limited, as patient factors such as size and shape, as well as scanner factors such as fluctuations in KVp, will affect the absolute value. However, a constant ratio between the liver and spleen attenuation values has been demonstrated, with the spleen acting as an internal control or reference value, eradicating factors such as fluctuations in KVp. Comparison of liver attenuation to spleen attenuation (L/S ratio) has been demonstrated to limit errors of variation in CT attenuation numbers between

Table 2 Mean values of liver attenuation, liver-to-spleen ratio and biochemical markers in the patient cohort. The liver attenuation, in Hounsfield units, the liver-to-spleen ratio (L/S ratio) and the measured biochemical markers are tabulated for comparison between the whole cohort, and the female and male patients.

\begin{tabular}{|c|c|c|c|c|c|c|}
\hline & \multicolumn{2}{|c|}{ All patients } & \multicolumn{2}{|c|}{ Female patients } & \multicolumn{2}{|c|}{ Male patients } \\
\hline & No. & Mean (S.D.) & No. & Mean (S.D.) & No. & Mean (S.D.) \\
\hline Liver attenuation & 51 & $50.8(14)$ & 42 & $51.8(14.9)$ & 9 & $46.4(9.7)$ \\
\hline L/S ratio & 50 & $1.17(0.31)$ & 42 & $1.17(0.31)$ & 8 & $1.19(0.29)$ \\
\hline Mean serum cortisol & 47 & $638.9(335.0)$ & 38 & $602.8(325.0)$ & 9 & $791.4(350.6)$ \\
\hline Mean ACTH & 46 & $44.6(37.8)$ & 37 & $38.9(35.5)^{\star}$ & 9 & $67.9(40.0)^{\star}$ \\
\hline Cholesterol & 21 & $6.2(1.3)$ & 19 & $6.16(1.3)$ & 2 & $6.6(2.0)$ \\
\hline Triglycerides & 20 & $2.1(1.1)$ & 18 & $2.2(1.1)$ & 2 & $1.2(0.61)$ \\
\hline LDL & 11 & $4.6(1.2)$ & 10 & 4.5 (1.3) & 1 & 5.5 \\
\hline $\mathrm{HDL}$ & 11 & $1.2(0.3)$ & 10 & $1.1(0.2)$ & 1 & 1.8 \\
\hline ALP & 26 & $102.6(110.7)$ & 22 & 104.9 (119.3) & 4 & $90.3(46.7)$ \\
\hline AST & 25 & $21.9(10.8)$ & 20 & $20.8(11.0)$ & 5 & $26.4(9.7)$ \\
\hline ALT & 19 & $35.8(20.5)$ & 15 & $32.3(13.5)$ & 4 & $48.8(37.2)$ \\
\hline GGT & 10 & $117.1(119.6)$ & 7 & $72.6(67.6)$ & 3 & $221.0(165.8)$ \\
\hline Bilirubin & 26 & $8.1(3.6)$ & 21 & $7.7(2.7)$ & 5 & $10.0(6.3)$ \\
\hline
\end{tabular}

* Confidence interval analysis for the comparison of means demonstrates only one significant difference at the $95 \%$ level, between the mean ACTH levels of the male and female patients. No other significant difference is found between the male and female patients. LDL, low density lipoproteins; HDL, high density lipoproteins; ACTH, adrenocorticotrophic hormone; ALP, alkaline phosphatase; AST, asparate transminase, ALT, alanine transaminase; GGT, gamma-glutamyl transaminase. 
Table 3 Spearman's correlation table of hepatic steatosis and biochemical markers of liver disease and Cushing's syndrome. The Spearman's correlation coefficient (rho) and the significance level $(P)$ for the liver attenuation in Hounsfield units (liver $\mathrm{HU}$ ) and the liver-to-spleen attenuation ratio (L/S ratio) of various biochemical markers are shown together with the number of patients $(n)$.

\begin{tabular}{|c|c|c|c|}
\hline & & Liver HU & L/S ratio \\
\hline \multirow[t]{3}{*}{ CDC } & rho & -0.121 & -0.035 \\
\hline & $P$ & 0.430 & 0.823 \\
\hline & $n$ & 45 & 44 \\
\hline \multirow[t]{3}{*}{ ACTH mean } & rho & -0.095 & 0.036 \\
\hline & $P$ & 0.540 & 0.820 \\
\hline & $n$ & 44 & 43 \\
\hline \multirow[t]{3}{*}{ Cholesterol } & rho & -0.178 & -0.069 \\
\hline & $P$ & 0.452 & 0.773 \\
\hline & $n$ & 20 & 20 \\
\hline \multirow[t]{3}{*}{ TG } & rho & -0.211 & -0.186 \\
\hline & $P$ & 0.385 & 0.446 \\
\hline & $n$ & 19 & 19 \\
\hline \multirow[t]{3}{*}{ LDL } & rho & 0.064 & -0.213 \\
\hline & $P$ & 0.861 & 0.554 \\
\hline & $n$ & 10 & 10 \\
\hline \multirow[t]{3}{*}{ HDL } & rho & 0.043 & 0.357 \\
\hline & $P$ & 0.907 & 0.312 \\
\hline & $n$ & 10 & 10 \\
\hline \multirow[t]{3}{*}{ ALP } & rho & 0.311 & 0.423 \\
\hline & $P$ & 0.140 & $0.044^{\star}$ \\
\hline & $n$ & 24 & 23 \\
\hline \multirow[t]{3}{*}{ AST } & rho & 0.099 & 0.183 \\
\hline & $P$ & 0.661 & 0.427 \\
\hline & $n$ & 22 & 21 \\
\hline \multirow[t]{3}{*}{ ALT } & rho & -0.296 & 0.224 \\
\hline & $P$ & 0.248 & 0.388 \\
\hline & $n$ & 17 & 17 \\
\hline \multirow[t]{3}{*}{ GGT } & rho & 0.287 & 0.544 \\
\hline & $P$ & 0.422 & 0.130 \\
\hline & $n$ & 10 & 9 \\
\hline \multirow[t]{3}{*}{ BIL } & rho & 0.073 & -0.011 \\
\hline & $P$ & 0.740 & 0.962 \\
\hline & $n$ & 23 & 22 \\
\hline
\end{tabular}

${ }^{\star} P<0.05$ (2-tailed).

CDC, cortisol day-curve mean. ACTH, adrenocorticotrophic hormone. Fasting lipids: TG, triglycerides; HDL, high density lipoproteins: LDL, low density lipoproteins. ALP, alkaline phosphatase; AST, aspartate transminase; $\mathrm{ALT}$, alanine transaminase; GGT, gamma-glutamyl transaminase; BIC, bilirubin.

scanners or with time on the same scanner. The L/S ratio is a reliable way of assessing fatty infiltration of the liver $(9,10)$.

The CT prevalence of hepatic steatosis has been documented in a large retrospective study of unselected hospital patients in Saudi Arabia (17). A prevalence of $9.7 \%$ was identified, using an absolute attenuation value of -10 Hounsfield units as the definition of hepatic steatosis. The choice of a very low attenuation value was used in order to identify only those patients in whom the liver was almost certainly steatotic. Unfortunately, the L/S ratio was not calculated in this study. There is no study in the published literature quoting the prevalence of hepatic steatosis in an unselected group of patients using the now accepted L/S ratio $<1$ as the definition of hepatic steatosis.

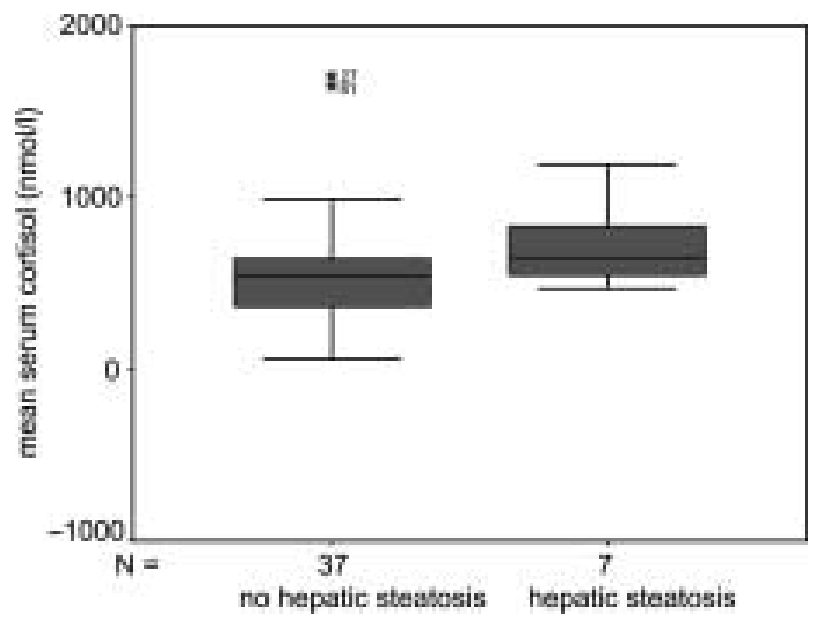

Figure 3 Box-and-whisker plot of the mean serum cortisol levels from the cortisol day curve in patients with no hepatic steatosis and in patients with hepatic steatosis (defined as an L/S ratio of less than 1). The box-and-whisker plot shows the 2.5, 25, 50 (the median), 75 and $97.5 \%$ cumulative relative frequencies (centiles). Cases 27 and 61 were outliers.

Table 4 Spearman's correlation table of liver attenuation and L/S ratio with age and body fat measurements. The Spearman's correlation coefficient (rho) and the significance level $(P)$ for the liver attenuation in Hounsfield units (liver HU) and the liver-tospleen attenuation ratio (L/S ratio) of age and body fat measurements are shown together with the number of patients $(n)$.

\begin{tabular}{lccc}
\hline & & Liver HU & L/S ratio \\
\hline Age & rho & -0.184 & -0.227 \\
& $P$ & 0.196 & 0.113 \\
Weight & $n$ & 51 & 50 \\
& rho & -0.170 & -0.221 \\
BMI & $P$ & 0.294 & 0.171 \\
& $n$ & 40 & 40 \\
Total fat & rho & -0.208 & -0.206 \\
& $P$ & 0.270 & 0.276 \\
Visceral fat & $n$ & 30 & 30 \\
& rho & -0.441 & -0.526 \\
Subcut fat & $P$ & $0.021^{*}$ & $0.006 *$ \\
& $n$ & 27 & 26 \\
\% V fat & $P$ & -0.616 & -0.638 \\
& $n$ & $0.001^{* *}$ & $0.000^{* *}$ \\
& rho & 27 & 26 \\
V: S ratio & $P$ & -0.174 & -0.283 \\
& $n$ & 0.386 & 0.161 \\
& rho & 27 & 26 \\
& $P$ & -0.613 & -0.464 \\
& $n$ & $0.001^{* *}$ & $0.017^{*}$ \\
& rho & 27 & 26 \\
& $P$ & 0.613 & -0.464 \\
& $n$ & $0.001^{* *}$ & $0.017^{*}$ \\
& & 27 & 26 \\
\hline
\end{tabular}

${ }^{\star} P<0.05$ level (2-tailed), ${ }^{* \star} P<0.001$ level (2-tailed).

$\mathrm{BMI}$, body mass index; subcut, subcutaneous fat area; $\% \mathrm{~V}$ fat, percentage of visceral to total fat area; $\mathrm{V}: \mathrm{S}$, visceral to subcutaneous fat area.

In our study, hepatic steatosis occurred in $20 \%$ of patients using a CT diagnosis of $\mathrm{L} / \mathrm{S}$ ratio of less than one. Unfortunately, there are no control data available for eucortisolaemic patients matched for age, sex and 
BMI, and it was not considered ethically possible to recruit such a group due to the radiation dose associated with CT scanning. The prevalence of hepatic steatosis in our group of patients with Cushing's syndrome appears to be greater than the values found in the literature in non-selected patients, on ultrasound and on $\mathrm{CT}$, but direct comparison with such varying data was not considered appropriate.

Our data demonstrated a strong negative correlation between the presence of hepatic steatosis and an increase in the total and visceral fat area calculated on CT but not the subcutaneous fat area. The relationship between increased visceral fat and hepatic steatosis has been considered previously in the literature, in eucortisolaemic patients, using anthropometric techniques rather than CT. A strong positive correlation has been found between the waist-to-hip ratio and hepatic steatosis in obese patients $(19,20)$. There has been no previously published study to our knowledge demonstrating a correlation between CT body fat measurements and hepatic steatosis in eucortisolaemic or cushingoid patients.

The finding of non-alcoholic fatty liver and NASH in patients with simple obesity and abnormal liver function tests is relatively common, with an incidence of increased fibrosis or cirrhosis of $15-50 \%$, and in very obese patients the finding of chronic liver disease histologically indistinguishable from alcoholic liver disease is well recognised (21). The mechanism for this is uncertain, but may be related to the increased flux of free fatty acids from the visceral fat into the liver via the portal vein (22). Thus, in Cushing's syndrome, it may be the increase in visceral fat which results in hepatic steatosis rather than a direct effect of cortisol on the liver itself. However, it is perhaps noteworthy that our patients' mean BMI levels were not very high, reflecting an 'overweight' state rather than profoundly 'obese'. The reversibility of the changes shown in Cushing's syndrome with its successful treatment and alternatively the incidence of any progression to NASH is not yet established.

In conclusion, we have documented the presence of hepatic steatosis in $20 \%$ of patients with active Cushing's syndrome using CT. We have also demonstrated a strong correlation between the presence of increased visceral fat and hepatic steatosis as measured by CT, which has not previously been documented. This finding may have important implications, with a potential for an increase in NASH in this group of patients.

\section{References}

1 Byron D \& Minuk GY. Clinical hepatology: profile of an urban, hospital-based practice. Hepatology 199624 813-815.

2 Saadeh S \& Younossi ZM. The spectrum of nonalcoholic fatty liver disease: from steatosis to nonalcoholic steatohepatitis. Cleveland Clinic Journal of Medicine 200067 96-97.
3 Falck-Ytter Y, Younossi ZM, Marchesini G \& McCullough AJ. Clinical features and natural history of nonalcoholic steatosis syndromes. Seminars in Liver Disease 200121 17-26.

4 Ludwig J, Viggiano TR, McGill DB \& Oh BJ. Nonalcoholic steatohepatitis: Mayo Clinic experiences with a hitherto unnamed disease. Mayo Clinic Proceedings 198055 434-438.

5 Teli MR, James OF, Burt AD, Bennett MK \& Day CP. The natural history of nonalcoholic fatty liver: a follow-up study. Hepatology $1995221714-1719$.

6 Matteoni CA, Younossi ZM, Gramlich T, Boparai N, Liu YC \& McCullough AJ. Nonalcoholic fatty liver disease: a spectrum of clinical and pathological severity. Gastroenterology 1999116 1413-1419.

7 James OF \& Day CP. Non-alcoholic steatohepatitis (NASH): a disease of emerging identity and importance. Journal of Hepatology 199829 495-501.

8 Bydder GM, Chapman RW, Harry D, Bassan L, Sherlock S \& Kreel L. Computed tomography attenuation values in fatty liver. Journal of Computed Tomography 19815 33-35.

9 Ricci C, Longo R, Gioulis E, Bosco M, Pollesello P, Masutti F et al. Noninvasive in vivo quantitative assessment of fat content in human liver. Journal of Hepatology 199727 108-113.

10 Piekarski J, Goldberg HI, Royal SA, Axel L \& Moss AA. Difference between liver and spleen CT numbers in the normal adult: its usefulness in predicting the presence of diffuse liver disease. Radiology $1980137727-729$.

11 Newell-Price J, Trainer P, Besser M \& Grossman A. The diagnosis and differential diagnosis of Cushing's syndrome and pseudoCushing's states. Endocrine Reviews $199819647-672$.

12 Borkan GA, Gerzof SG, Robbins AH, Hults DE, Silbert CK \& Silbert JE. Assessment of abdominal fat content by computed tomography. American Journal of Clinical Nutrition 198236 $172-177$.

13 Hilden M, Christoffersen P, Juhl E \& Dalgaard JB. Liver histology in a 'normal' population - examinations of 503 consecutive fatal traffic casualties. Scandinavian Journal of Gastroenterology 1977 12 593-597.

14 Ground KE. Liver pathology in aircrew. Aviation, Space and Environmental Medicine 198253 14-18.

15 Tsushima Y \& Endo K. Spleen enlargement in patients with nonalcoholic fatty liver: correlation between degree of fatty infiltration in liver and size of spleen. Digestive Diseases and Sciences 200045 196-200.

16 Nomura H, Kashiwagi S, Hayashi J, Kajiyama W, Tani S \& Goto M. Prevalence of fatty liver in a general population of Okinawa, Japan. Japanese Journal of Medicine 198827 142-149.

17 el Hassan AY, Ibrahim EM, al Mulhim FA, Nabhan AA \& Chammas MY. Fatty infiltration of the liver: analysis of prevalence, radiological and clinical features and influence on patient management. British Journal of Radiology $1992 \mathbf{6 5}$ $774-778$.

18 Bellentani S, Tiribelli C, Saccoccio G, Sodde M, Fratti N, De Martin C et al. Prevalence of chronic liver disease in the general population of northern Italy: the Dionysos Study. Hepatology $1994201442-1449$.

19 Kral JG, Schaffner F, Pierson RN Jr \& Wang J. Body fat topography as an independent predictor of fatty liver. Metabolism $1993 \mathbf{4 2}$ 548-551.

20 Lonardo A \& Trande P. Are there any sex differences in fatty liver? A study of glucose metabolism and body fat distribution. Journal of Gastroenterology and Hepatology $2000 \mathbf{1 5}$ 775-782.

21 James O \& Day C. Non-alcoholic steatohepatitis: another disease of affluence. Lancet 1999353 1634-1636.

22 Chitturi S \& Farrell GC. Etiopathogenesis of nonalcoholic steatohepatitis. Seminars in Liver Disease $20012127-41$.

Received 15 April 2003

Accepted 22 August 2003 